\title{
Coupling of temperature with pressure induced initial decomposition mechanisms of two molecular crystals: An ab initio molecular dynamics study
}

\author{
QIONG WU, ${ }^{\mathrm{a}, \mathrm{b}}$, DONG XIANG ${ }^{\mathrm{a}}$, GUOLIN XIONG ${ }^{\mathrm{a}}$, WEIHUA ZHU ${ }^{\mathrm{a}, *}$ and HEMING XIAO ${ }^{\mathrm{a}}$ \\ anstitute for Computation in Molecular and Materials Science and Department of Chemistry, School of \\ Chemical Engineering, Nanjing University of Science and Technology, Nanjing 210094, China \\ ${ }^{\mathrm{b}}$ School of Materials Science and Engineering, Nanjing Institute of Technology, Nanjing 211167, China \\ e-mail: zhuwh@njust.edu.cn
}

MS received 12 December 2015; revised 26 January 2016; accepted 24 February 2016

\begin{abstract}
Ab initio molecular dynamics simulations were performed to study the initiation of decomposition and formation of first products of two molecular crystals pentaerythritol tetranitrate (PETN) and 5-nitro-2,4dihydro-1,2,4-triazole-3-one (NTO) under thermal decomposition temperature (475 K for PETN and $531 \mathrm{~K}$ for NTO) coupled with different pressures (1-5 GPa). The pressure effects on the initial decomposition steps and initially generated products on PETN and NTO were very different. PETN was triggered by $\mathrm{C}-\mathrm{H} \cdots \mathrm{O}$ intermolecular hydrogen transfer. The initial decomposition mechanism was independent of the pressure. For NTO, two different initial decomposition mechanisms were found. At 1,2 , and $3 \mathrm{GPa}$, it was triggered by N$\mathrm{H} \cdots \mathrm{O}$ intermolecular hydrogen transfer, while at 4 and $5 \mathrm{GPa}$, it was triggered by $\mathrm{N}-\mathrm{H} \cdots \mathrm{N}$ intermolecular hydrogen transfer. This indicates that the initial decomposition mechanism was dependent on the pressure. Our study may provide new insights into initial mechanisms and decomposition reactions of molecular crystal explosives under thermal decomposition temperature coupled with different pressures with details at atomic level.
\end{abstract}

Keywords. Ab initio molecular dynamics; Pentaerythritol tetranitrate; 5-Nitro-2,4-dihydro-1,2,4-triazole-3one; Initiation decomposition mechanism; high temperature; high pressure.

\section{Introduction}

Initial decomposition mechanisms of high explosives under coupled effects of temperature and pressure are important. Studies are necessary for understanding their complicated reactive behavior and guaranteeing the safety in practical production, storage, transportation and usage. Many high explosives are organic molecular crystals. Among them, pentaerythritol tetranitrate (PETN, figure 1) and 5-nitro-2,4-dihydro-1,2,4triazole-3-one (NTO, figure 1) are two important representatives. However, due to high complexity, difficulty, and hazards associated with such experiments, the initial decomposition mechanisms of PETN and NTO are far from being well understood until now. At present, theoretical methods like ab initio molecular dynamics (AIMD) may be an effective way for investigating the physical and chemical properties of the high explosives under coupled effects of temperature and pressure.

PETN,$^{1-3}$ a powerful secondary high explosive, is the most stable and least reactive nitric ester explosive

*For correspondence till now and widely used in commercial blasting caps, detonation cords, and boosters. Its thermal decomposition temperature is $475 \mathrm{~K}$. Two experimental ${ }^{4,5}$ studies have investigated its structure and decomposition around this temperature coupled with high pressures up to 14 $\mathrm{GPa}$. The first study ${ }^{4}$ reported that the high temperaturepressure phase diagram includes the melting/decomposition curve and boundaries between the three PETN phases. Another one ${ }^{5}$ found that its initial decomposition step is a bimolecular type. However, both of them did not give the detailed initial mechanism and first decomposition product of PETN.

$\mathrm{NTO}^{6-8}$ is a high energy but very insensitive explosive and has been widely used in explosive formulations, PBXs (polymer-bonded explosives), and generators. Its thermal decomposition temperature is about $531 \mathrm{~K}$, but few experimental and theoretical studies have been devoted to investigate its structure and decomposition around this temperature coupled with high pressure. Its initial decomposition steps under this condition are unknown still and so related research are necessary. 


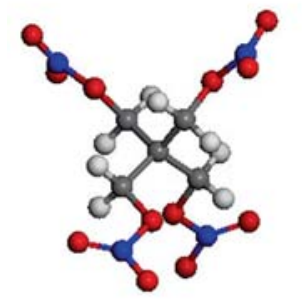

(a) molecule

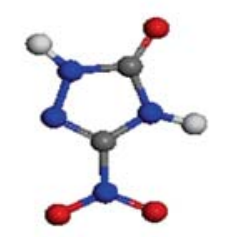

(b) molecule

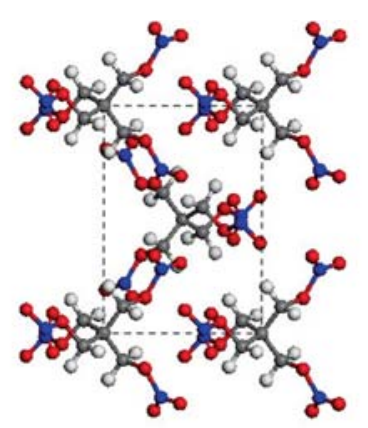

unit cell

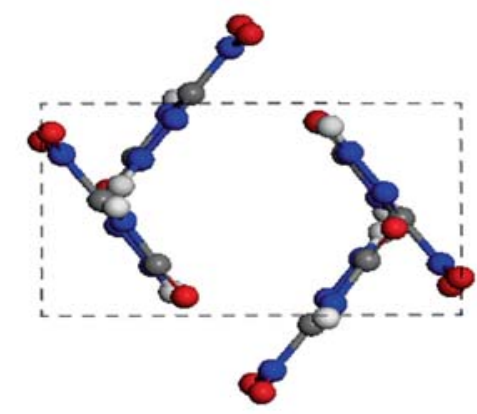

unit cell

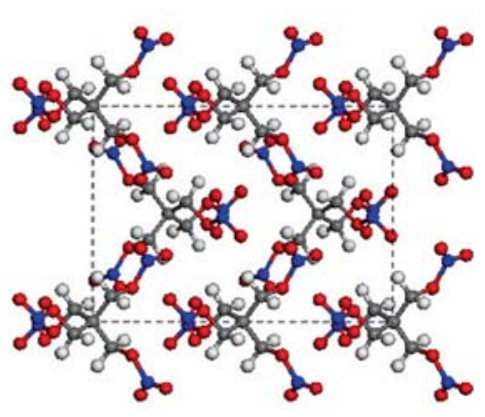

$1 \times 1 \times 2$ supercell

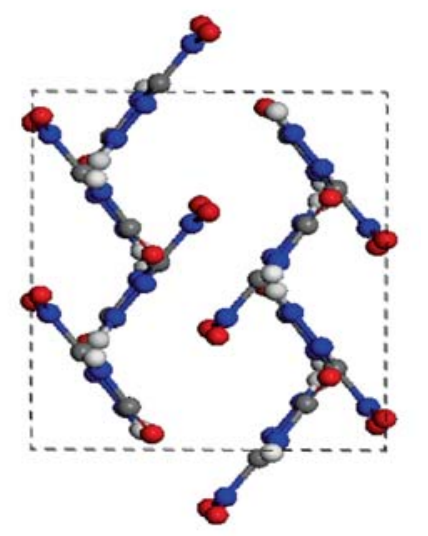

$1 \times 2 \times 1$ supercell

Figure 1. The molecule, unit cells, and supercells of (a) PETN and (b) NTO. Carbon, nitrogen, oxygen, and hydrogen atoms are represented by gray, blue, red and white spheres, respectively.

Table 1. Comparison of experimental and relaxed cell parameters, space group, and crystal system of PETN crystal at ambient conditions.

\begin{tabular}{lcccccc}
\hline Parameter & $a(\AA)$ & $b(\AA)$ & $c(\AA)$ & Volume $\left(\AA^{3}\right)$ & Space group & Crystal system \\
\hline Expt. $^{1}$ & 9.276 & 9.276 & 6.613 & 569.0 & $P 42_{1} c$ & Tetragonal \\
PBE & 9.876 & 9.876 & 6.860 & 669.1 & $P 42_{1} c$ & Tetragonal \\
PBE-TS & 9.259 & 9.259 & 6.646 & 569.8 & $P 42_{1} c$ & Tetragonal \\
PBE-G06 & 9.219 & 9.219 & 6.684 & 568.1 & $P 42_{1} c$ & Tetragonal \\
\hline
\end{tabular}

In this work, we performed high-level AIMD simulations to study the initiation decomposition mechanisms of two high explosives PETN and NTO under thermal decomposition temperature $(475 \mathrm{~K}$ for PETN and $531 \mathrm{~K}$ for NTO) coupled with different pressures (1-5 GPa). Our main purpose is to determine how the pressure affects the decomposition of PETN and NTO under thermal decomposition conditions.

\section{Computational method}

Our MD simulations were performed within the framework of DFT based on CASTEP code $^{9}$ using norm-conserving pseudopotentials ${ }^{10}$ and a plane-wave expansion of the wave functions. The Perdew-BurkeErnzerhof $(\mathrm{PBE})^{11}$ exchange-correlation function and a single k-point were employed. We utilized a plane wave cutoff of $500 \mathrm{eV}$ for MD simulations and of $830 \mathrm{eV}$ for geometry and cell optimizations. To correct DFT for missing van der Waals (vdW) interaction, we used Tkatchenoko and Scheffer (TS $)^{12}$ and Grimme $(\mathrm{G} 06)^{13}$ corrections to the PBE functional (labeled as PBE-TS and PBE-G06), respectively. Table 1 lists the experimental and relaxed cell parameters of the PETN crystal. It is found that the results by PBE without the vdW correction are obviously higher than the experimental results, especially for the lattice parameters $a$ and $b$. However, this inconsistency can be amended when proper vdW correction is considered. For instance, the 
calculated $c$ by the PBE-TS and PBE-G06 methods are very close to the experimental value, indicating that the $\mathrm{vdW}$ correction is necessary for investigating the PETN crystal. In all, the estimated results by the PBE-TS method are in best agreement with the experiment values. Thus, we employed the PBE-TS method to investigate the structure and decomposition of PETN under thermal decomposition temperature $(475 \mathrm{~K})$ coupled with different pressures (1-5 GPa).

Table 2 lists the experimental and relaxed cell parameters of the NTO crystal. It is found that the results by PBE without the vdW correction are either lower or higher obviously than the experimental values, especially for the lattice parameters $b$ and $c$. However, this inconsistency can be well corrected when PBE is combined with proper vdW corrections. For example, the calculated $c$ by the PBE-TS and PBE-G06 methods are very close to the experimental value, which indicates that the $\mathrm{vdW}$ correction is needed for studying the NTO crystal. In all, the estimated results by the PBETS method are in the best agreement with the experiment values. Thus, we employed the PBE-TS method to investigate the structure and decomposition of NTO under thermal decomposition temperature $(531 \mathrm{~K})$ with different pressures (1-5 GPa).

We controlled the ionic temperature and pressure using a Nosé thermostat ${ }^{14}$ and an Andersen barostat ${ }^{15}$ respectively. A time step of $1.0 \mathrm{fs}$ was used in time integration. Previous studies ${ }^{16}$ reported that a time step of 1.2 fs was used to study the thermal decomposition of the highly explosive PETN. A time step of 1.0 fs was also adopted in the study of iron ${ }^{17}$ by using AIMD. Both the NVT and NPT ensembles were employed. The initial positions of the simulation supercells were taken from the experimentally determined X-ray crystal structure. ${ }^{1,6}$ We used bond-length criteria to identify the changes in geometry during the simulations. Considering the quite long simulation time and high cost, the decomposition simulations of PETN and NTO were first performed on a $1 \times 1 \times 2$ supercell ( 4 molecules and 116 atoms, figure 1 ) and a $1 \times 2 \times 1$ supercell ( 8 molecules and 88 atoms, figure 1) respectively. First, the system was equilibrated at $298.15 \mathrm{~K}$ for $5 \mathrm{ps}$ using NVT. Then, based on this equilibrated system, for
PETN, at $475 \mathrm{~K}$ (thermal decomposition temperature), AIMD simulations were carried out using NPT at 1, 2, 3 , 4, and $5 \mathrm{GPa}$, respectively. While for NTO, at 531 $\mathrm{K}$ (thermal decomposition temperature), AIMD simulations were carried out using NPT at 1,2, 3, 4, and 5 $\mathrm{GPa}$, respectively. The simulation time is $20 \mathrm{ps}$ for all the AIMD simulations. We carried out 5 independent simulations on the decompositions of PETN and NTO, respectively.

\section{Results and Discussion}

\subsection{PETN}

3.1a Crystal Structure: Figure 2 displays the pressure dependence of the lattice parameters and volumes of PETN at the thermal decomposition temperature of $475 \mathrm{~K}$. It is seen that the changing trends in the lattice parameters are very similar, the lattice parameters decrease monotonously with the increasing pressure, especially when the pressure increases from 1 to $2 \mathrm{GPa}$. The PETN system is compressed by about $54 \%$ when the pressure increases from 1 to $5 \mathrm{GPa}$, indicating that the crystal structure of PETN is sensitive to compression at thermal decomposition temperature.

3.1b Initial decomposition mechanism: Previous theoretical study ${ }^{18}$ reported the energy barriers of several possible initial decomposition reactions for PETN at $0 \mathrm{~K}$ and found that the $\mathrm{O}-\mathrm{NO}_{2}$ cleavage has the lowest activation barriers. Another experimental study ${ }^{5}$ found that the initial decomposition of PETN around thermal decomposition temperature at different pressures was triggered by a bimolecular mechanism. This mechanism was presumed to eliminate $\mathrm{HNO}_{3}$, but this study did not give any experimental evidence. In the present study, we also observed the bimolecular initial decomposition reaction but did not find the elimination of $\mathrm{HNO}_{3}$. Table 3 lists the initial decomposition step of PETN at thermal decomposition temperature under different pressures. It is seen that under the pressures investigated except for $1 \mathrm{GPa}$, the initial decomposition step of PETN was triggered by $\mathrm{C}-\mathrm{H}$... O hydrogen

Table 2. Comparison of experimental and relaxed cell parameters, space group, and crystal system of NTO crystal at ambient conditions.

\begin{tabular}{lcccccc}
\hline Parameter & $a(\AA)$ & $b(\AA)$ & $c(\AA)$ & Volume $\left(\AA^{3}\right)$ & Space group & Crystal system \\
\hline Expt. $^{6}$ & 9.321 & 5.522 & 9.110 & 460.4 & $P 2_{1} / \mathrm{c}$ & monoclinic \\
PBE & 9.284 & 5.749 & 9.962 & 524.6 & $P 2_{1} / \mathrm{c}$ & monoclinic \\
PBE-TS & 9.241 & 5.590 & 9.127 & 464.6 & $P 2_{1} / \mathrm{c}$ & monoclinic \\
PBE-G06 & 9.199 & 5.588 & 9.018 & 456.2 & $P 2_{1} / \mathrm{c}$ & monoclinic \\
\hline
\end{tabular}



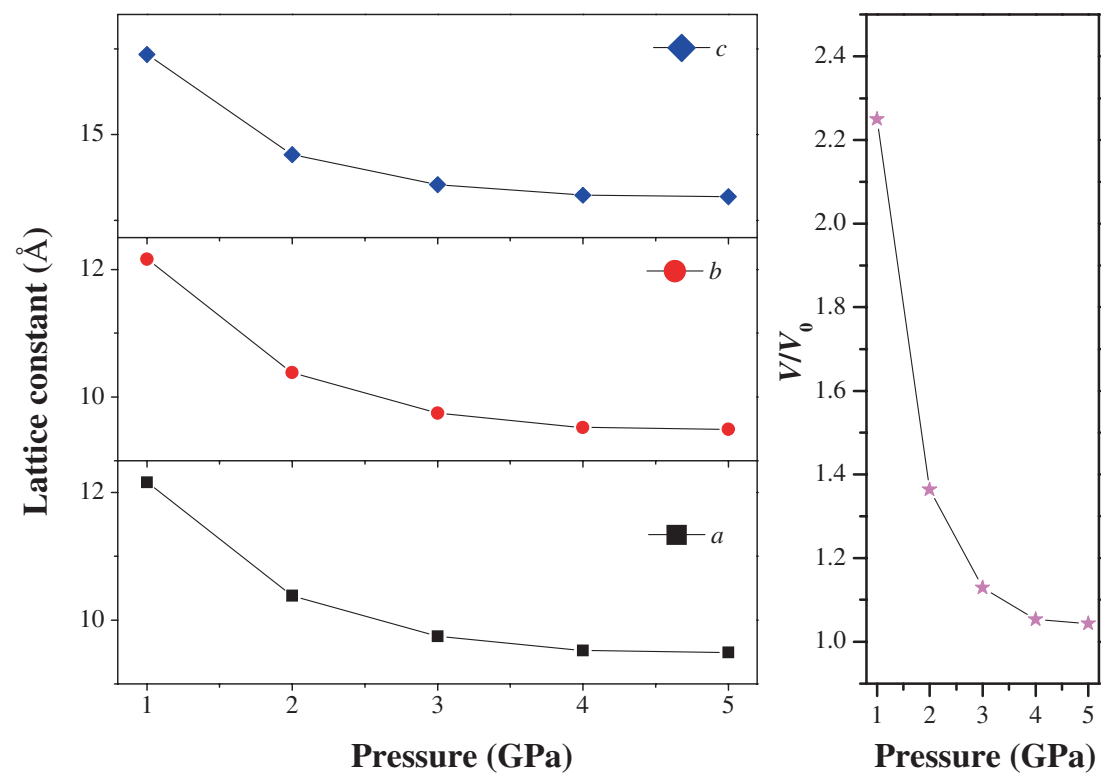

Figure 2. The pressure dependence of the lattice parameters and volumes of PETN at thermal decomposition temperature. $V_{0}$ is the volume of a $1 \times 1 \times 2$ supercell for PETN at ambient $(\mathrm{T}=298.15 \mathrm{~K}, \mathrm{P}=100 \mathrm{KPa})$ conditions.

Table 3. The initial decomposition step and first product of PETN under thermal decomposition temperature at different pressures.

\begin{tabular}{lcc}
\hline$P(\mathrm{GPa})$ & Initial decomposition step & First product \\
\hline 1 & melted without decomposing & melted without decomposing \\
2 & $\mathrm{C} \cdots \mathrm{H} \cdots$ O intermolecular hydrogen transfer & $\mathrm{NO}_{2}$ \\
3 & $\mathrm{C} \cdots \mathrm{H} \cdots$ O intermolecular hydrogen transfer & $\mathrm{NO}_{2}$ \\
4 & $\mathrm{C} \cdots \mathrm{H} \cdots$ O intermolecular hydrogen transfer & $\mathrm{NO}_{2}$ \\
5 & $\mathrm{C} \cdots \mathrm{H} \cdots$ O intermolecular hydrogen transfer & $\mathrm{NO}_{2}$ \\
\hline
\end{tabular}

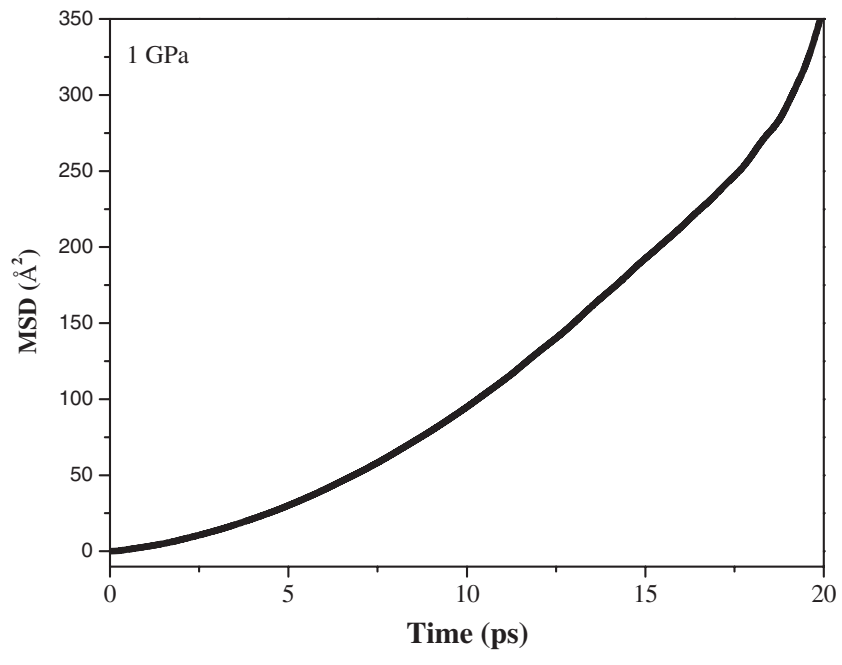

Figure 3. The calculated mean square displacement (MSD) of PETN under thermal ignition temperature at 1 GPa.

transfer between two molecules, which indicates that the initial decomposition of PETN at the pressure of 2-5 GPa under thermal decomposition temperature was independent of applied pressures. The energy barrier to this initial decomposition mechanism was calculated to be 78.3 and $78.7 \mathrm{kcal} / \mathrm{mol}$ at the B3LYP and M06-2X levels, respectively.

Figure 3 displays the calculated mean square displacement (MSD) of PETN under thermal decomposition temperature at $1 \mathrm{GPa}$. It is found that its MSD at $1 \mathrm{GPa}$ represents the characteristic of a liquid, indicating that PETN melted. In addition, at $1 \mathrm{GPa}$, no decomposition reactions were observed during the complete MD simulation of PETN, showing that PETN did not decompose. Thus, at $1 \mathrm{GPa}$, PETN melted without decomposition.

Figure 4 displays the snapshots before, during, and after the initiation decomposition of PETN. The intermolecular hydrogen transfer $(\mathrm{C}-\mathrm{H} \cdots \mathrm{O})$ can be observed clearly. First, one C-H bond length in one PETN molecule increased and the hydrogen atom in this $\mathrm{C}-\mathrm{H}$ bond tends to transfer to an oxygen atom of the nitro group in the adjacent PETN molecule. Then, the $\mathrm{C}-\mathrm{H}$ bond breaks and a new $\mathrm{O}-\mathrm{H}$ bond is formed, resulting in the formation of two intermediates (INT1 and INT2). 

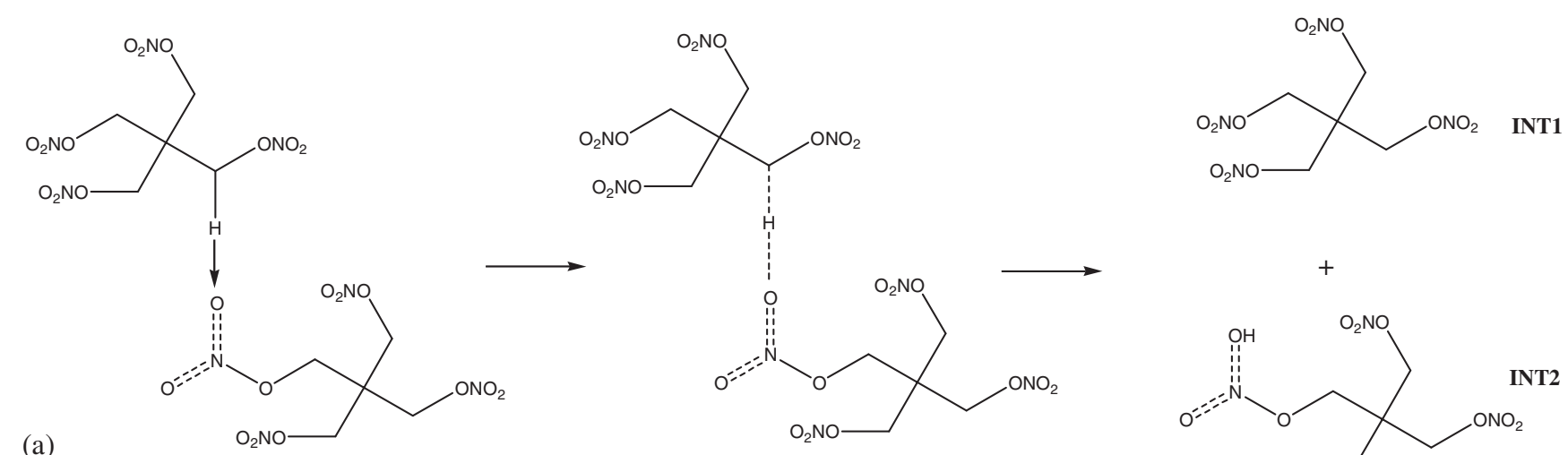

(a)

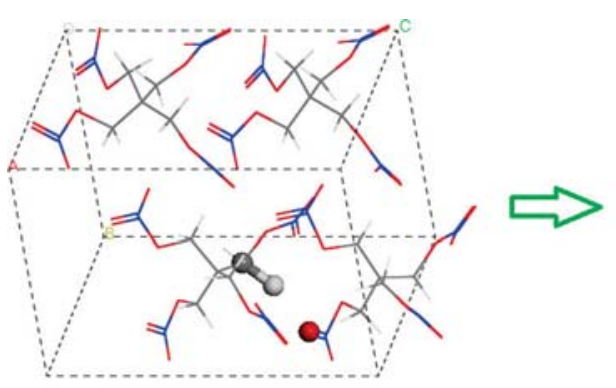

(b)

before

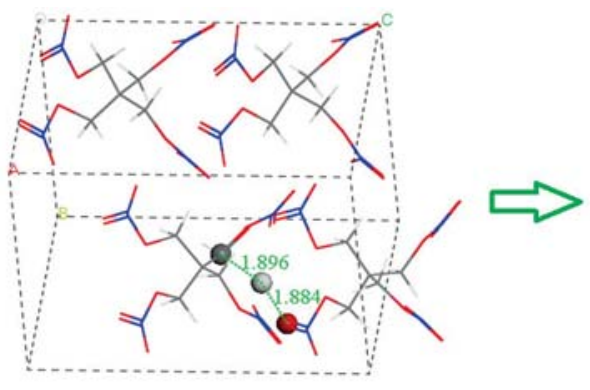

occurring

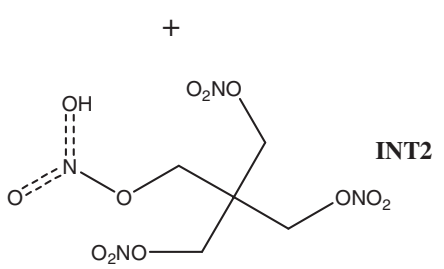

Figure 4. Snapshots before, during, and after initiation decomposition of PETN. Carbon, oxygen, and hydrogen atoms are represented by gray, red and white spheres, respectively.
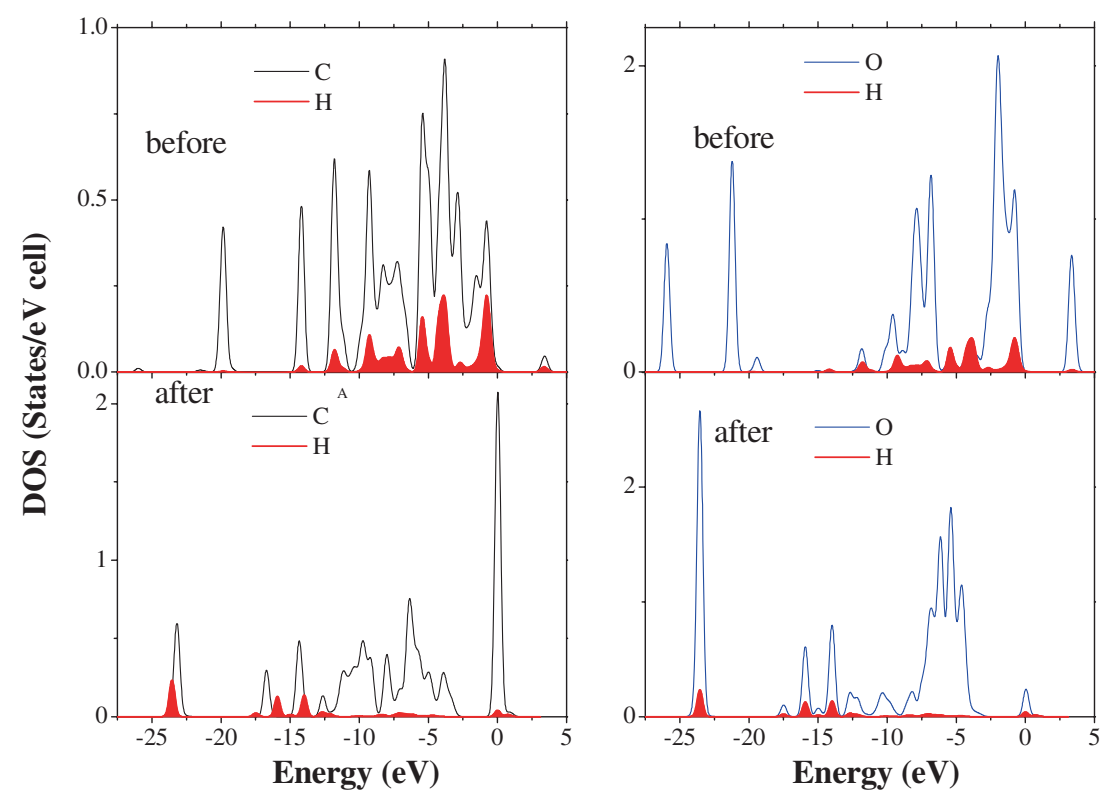

Figure 5. The changing process of the partial density of states (PDOS) of the $\mathrm{C}, \mathrm{H}$ and $\mathrm{O}$ atoms involved in the intermolecular hydrogen transfer in PETN crystal.

Based on the structures and reactions observed in the MD simulations, the density of states (DOS) of the atoms involved in the transfer reactions was calculated to provide further evidence and clarity for the breaking and formation process of the bonds. Figure 5 shows the changing process of the partial density of states (PDOS) of the $\mathrm{C}, \mathrm{O}$ and $\mathrm{H}$ atoms involved in the intermolecular hydrogen transfer. For the $\mathrm{C}$ and $\mathrm{H}$ atoms in one molecule, some strong DOS peaks occur at the same energy value just before the intermolecular 
hydrogen transfer, but after the hydrogen transfer, they are located at different energies. As for the $\mathrm{O}$ and $\mathrm{H}$ atoms in another molecule, the case is just the opposite; their strong peaks appear in different energy before initial decomposition, but after initial decomposition they occur at the same energy. These are due to the $\mathrm{C}-\mathrm{H}$ bond cleavage and the $\mathrm{O}-\mathrm{H}$ bond formation during the intermolecular hydrogen transfer. Before initial decomposition, the distance of $\mathrm{O}$ and $\mathrm{H}$ atoms are very large and they are not bonded. Thus, the bond order of the new $\mathrm{O}-\mathrm{H}$ bond is zero. After the decomposition, the new $\mathrm{O}-\mathrm{H}$ bond formed and their bond orders will increase significantly.

\subsection{Decomposition products}

The final decomposition products can be well identified by an experimental test. But, the generated first product after initial decomposition is usually unstable, making it very difficult to detect. Table 3 lists the first decomposition product of PETN under thermal decomposition temperature at different pressures. It is found that the first product is $\mathrm{NO}_{2}$ at all pressures except for $1 \mathrm{GPa}$, at which PETN only melted without further decomposition. The $\mathrm{NO}_{2}$ formed through the $\mathrm{C}(\mathrm{H}) \mathrm{O} \cdots \mathrm{NO}_{2}$ bond cleavage in INT1 and two selected snapshots of this process is shown in figure 6. The $\mathrm{NO}_{2}$ could further transform into NO through the reduction reaction $\mathrm{NO}_{2}+\mathrm{H}=\mathrm{NO}+\mathrm{OH}$. Due to low temperature of investigation $(475 \mathrm{~K})$, the degrees of the decomposition at all the pressures are low and only a few products and INTs formed. We did not observe $\mathrm{H}_{2} \mathrm{O}$ and $\mathrm{N}_{2} \mathrm{O}$.

To summarize, at thermal decomposition temperature, PETN melted without decomposition at $1 \mathrm{GPa}$, but it melted and decomposed at 2, 3, 4 and $5 \mathrm{GPa}$.
Its initial decomposition step was the hydrogen transfer (C-H... O) between two molecules. The first decomposition product is $\mathrm{NO}_{2}$. Both the initial decomposition mechanism and first product of PETN were independent of applied pressures.

\section{$3.3 \quad N T O$}

3.3a Crystal Structure: Figure 7 displays the pressure dependence of the lattice parameters and volumes of NTO at thermal decomposition temperature. It is seen that the changing trends of the three lattice parameters are similar: they decrease monotonously with the increasing pressure, especially when the pressure increases from 1 to $2 \mathrm{GPa}$. The NTO system is compressed to about $26 \%$ when the pressure increases from 1 to $5 \mathrm{GPa}$, indicating that the crystal structure of NTO is sensitive to applied pressures at thermal decomposition temperature.

3.3b Initial decomposition mechanism: Table 4 lists the initial decomposition step of NTO at thermal decomposition temperature coupled with different pressures. It is seen that at 1,2 and $3 \mathrm{GPa}$, the initial decomposition step of NTO was N-H. . O intermolecular hydrogen transfer, while at 4 and $5 \mathrm{GPa}$, NTO was triggered by $\mathrm{N}-\mathrm{H} \cdots \mathrm{N}$ intermolecular hydrogen transfer. This indicates that the initial decomposition mechanism of NTO in the pressure range of 1-5 GPa under thermal decomposition temperature was dependent on the pressure. In other words, the decomposition mechanism is affected by different applied pressures. This is different from that of PETN. By using the B3LYP and M06-2X methods, the energy barrier for the N-H. . O intermolecular hydrogen transfer was calculated to be
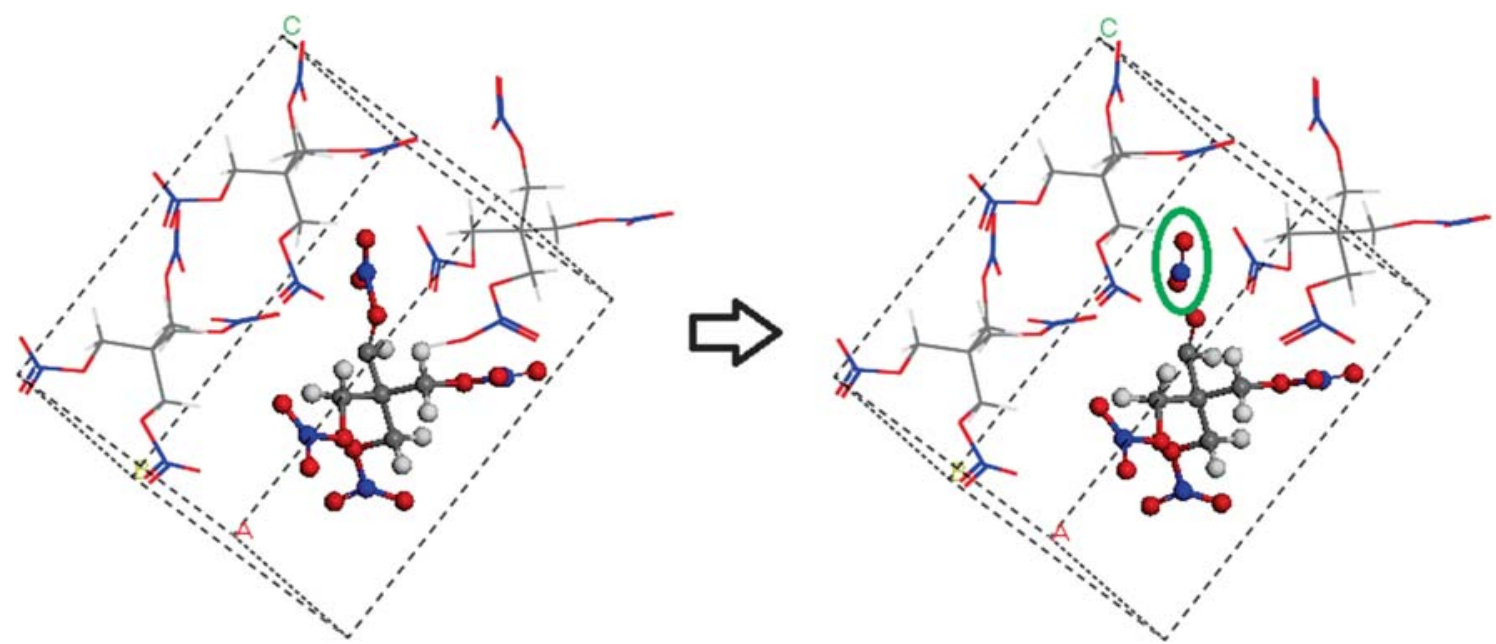

Figure 6. Selected snapshots of the formation process of first $\mathrm{NO}_{2}$ in PETN crystal. Carbon, nitrogen, oxygen, and hydrogen atoms are represented by gray, blue, red and white spheres, respectively. 

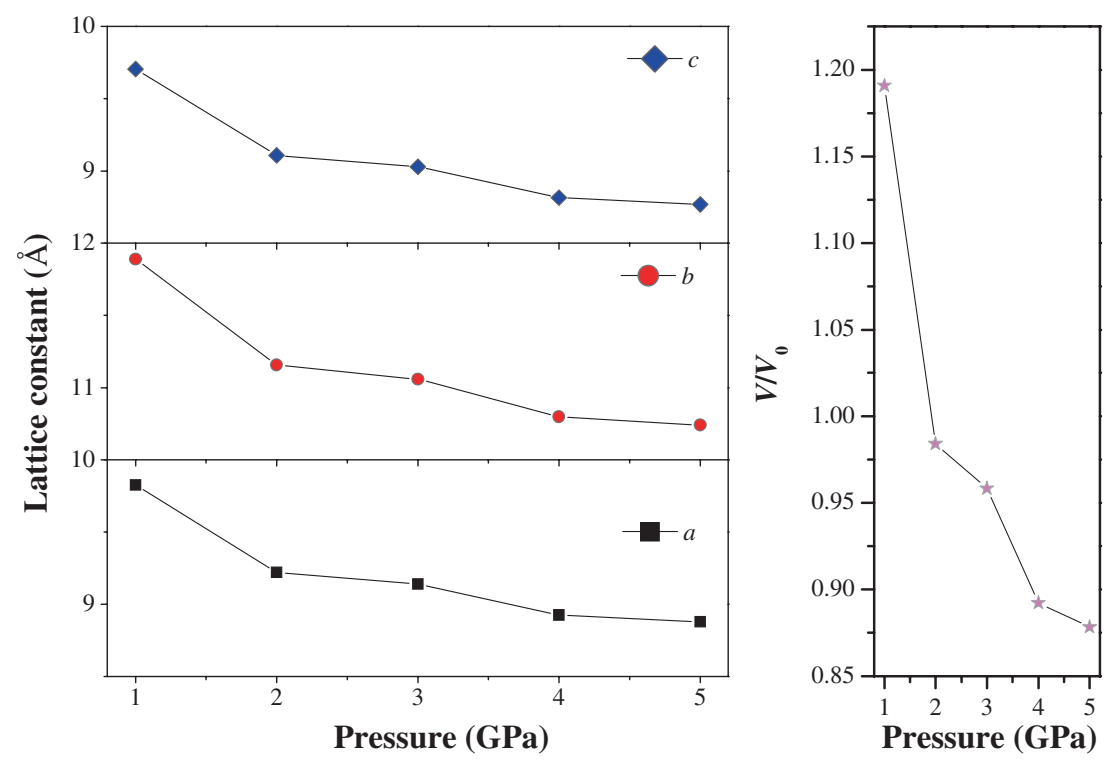

Figure 7. The pressure dependence of the lattice parameters and volumes of NTO at thermal decomposition temperature. $V_{0}$ is the volume of a $1 \times 2 \times 1$ supercell for NTO at ambient $(\mathrm{T}=298.15 \mathrm{~K}, \mathrm{P}=100 \mathrm{KPa})$ conditions.

Table 4. The initial decomposition step and first product of NTO under thermal decomposition temperature at different pressures.

\begin{tabular}{lcc}
\hline$P(\mathrm{GPa})$ & \multicolumn{1}{c}{ Initial decomposition step } & First product \\
\hline 1 & $\mathrm{~N} \cdots \mathrm{H} \cdots$ O type intermolecular hydrogen transfer & $\mathrm{N}_{2}$ \\
2 & $\mathrm{~N} \cdots \mathrm{H}$. type intermolecular hydrogen transfer & $\mathrm{N}_{2}$ \\
3 & $\mathrm{~N} \cdots \mathrm{H} \cdot \mathrm{O}$ type intermolecular hydrogen transfer & $\mathrm{H}_{2} \mathrm{O}$ \\
4 & $\mathrm{~N} \cdots \mathrm{H} \cdots \mathrm{N}$ type intermolecular hydrogen transfer & $\mathrm{NO}_{2}$ \\
5 & $\mathrm{~N} \cdots \mathrm{H} \cdots \mathrm{N}$ type intermolecular hydrogen transfer & $\mathrm{NO}_{2}$ \\
\hline
\end{tabular}

39.8 and $40.1 \mathrm{kcal} / \mathrm{mol}$ respectively, while the barrier for the $\mathrm{N}-\mathrm{H} \cdots \mathrm{N}$ intermolecular hydrogen transfer was 34.8 and $35.1 \mathrm{kcal} / \mathrm{mol}$, respectively.

Figure 8 shows the snapshots before, during and after the initiation of decomposition of NTO. The two different intermolecular hydrogen transfer processes can be observed clearly. For the bimolecular N-H...O intermolecular hydrogen transfer, the $\mathrm{N} 1-\mathrm{H}$ bond in one NTO molecule becomes longer and the hydrogen atom in this bond tends to transfer to the $\mathrm{O} 1$ atom of O1-C bond in an adjacent NTO molecule. Then, th $\mathrm{N} 1 \cdots \mathrm{H}$ bond breaks and a new $\mathrm{H}$. . O 1 bond is formed simultaneously generating two intermediates. As for the bimolecular $\mathrm{N}-\mathrm{H} \cdots \mathrm{N}$ intermolecular hydrogen transfer, the N1-H bond in one NTO molecule became longer and the hydrogen atom in this bond tends to transfer to the $\mathrm{N} 2$ atom in the ring of an adjacent NTO molecule. Then, this $\mathrm{N} 1-\mathrm{H}$ bond breaks and a new H-N2 bond is formed simultaneously generating two intermediates.

Figure 9 displays the changing process of the PDOS of the atoms involved in the $\mathrm{N}-\mathrm{H} \cdots \mathrm{O}$ and $\mathrm{N}-\mathrm{H} \cdot \cdots \mathrm{N}$ intermolecular hydrogen transfer respectively. In figure 9a, for the $\mathrm{N} 1$ and $\mathrm{H}$ atoms, some strong DOS peaks are observed with the same energy value just before the intermolecular hydrogen transfer, while those peaks occur at different energy after the hydrogen transfer. As for the $\mathrm{O} 1$ and $\mathrm{H}$ atoms, the case is just the opposite; their strong peaks appear at different energy value before initial decomposition, but after initial decomposition they occur at the same energy. These are due to the $\mathrm{N} 1-\mathrm{H}$ bond cleavage and the $\mathrm{O} 1-\mathrm{H}$ bond formation during the intermolecular hydrogen transfer. In figure $9 \mathrm{~b}$, for $\mathrm{N} 1$ and $\mathrm{H}$ atoms, their strong DOS peaks appear at the same energy value just before the intermolecular hydrogen transfer, but after the hydrogen transfer they appear with a different energy. The situation is just the opposite for the $\mathrm{O} 1$ and $\mathrm{H}$ atoms. These are caused by the $\mathrm{N} 1-\mathrm{H}$ bond cleavage and $\mathrm{H}-\mathrm{N} 2$ bond formation in the process of the $\mathrm{N}-\mathrm{H} \cdots \mathrm{N}$ intermolecular hydrogen transfer. Before initial decomposition, the distance of $\mathrm{N}$ and $\mathrm{H}$ atoms are very large and they are not bonded. Thus, the bond order of the new N-H bond is zero. After the decomposition, the new N-H bond formed and their bond orders will increase significantly. 
(a)<smiles></smiles><smiles>O=c1[nH]nc([N+](=O)[O-])[nH]c1=O</smiles>
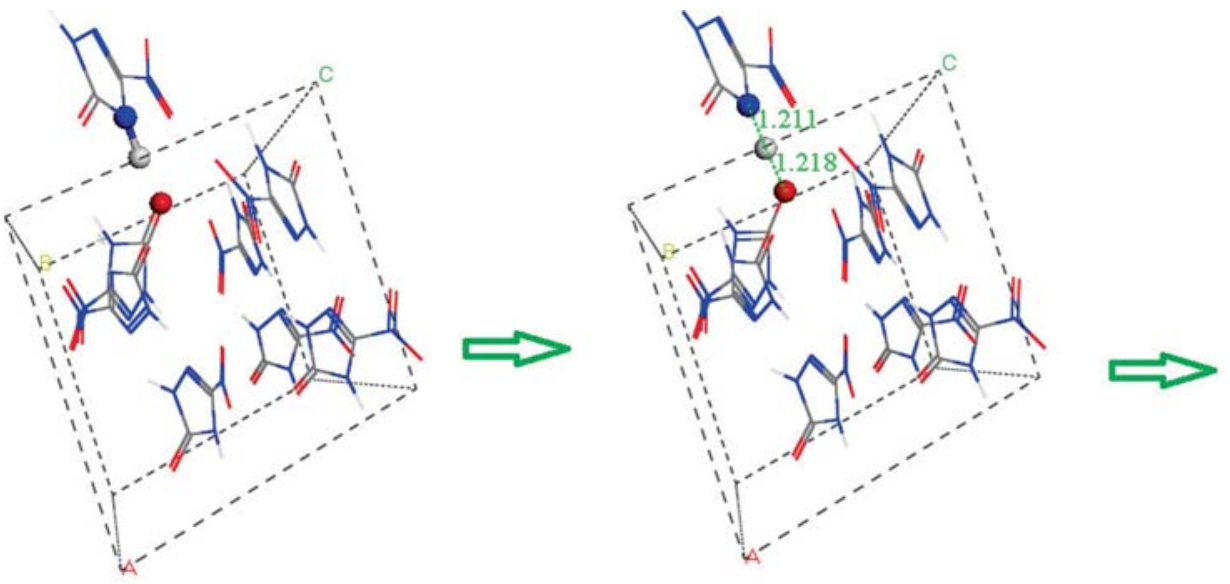

(b)

before

occurring

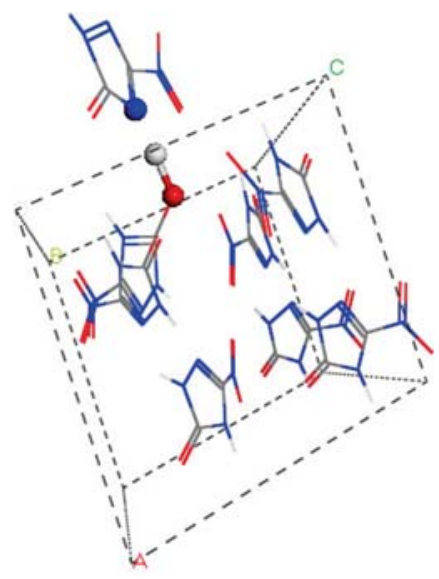

after

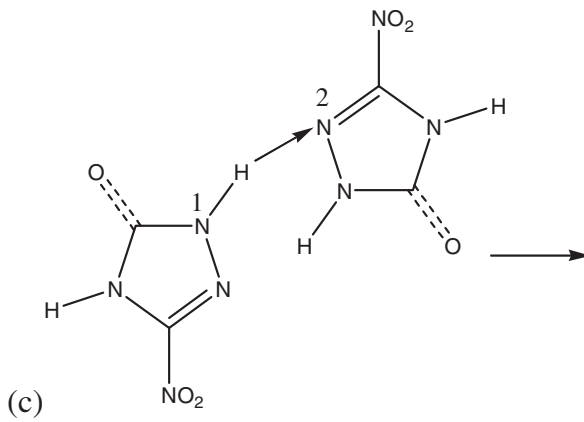<smiles>[2H]n1c([N+](=O)[O-])nn([AlH]c2nn(CC)c(=O)[nH]2)c1=O</smiles>

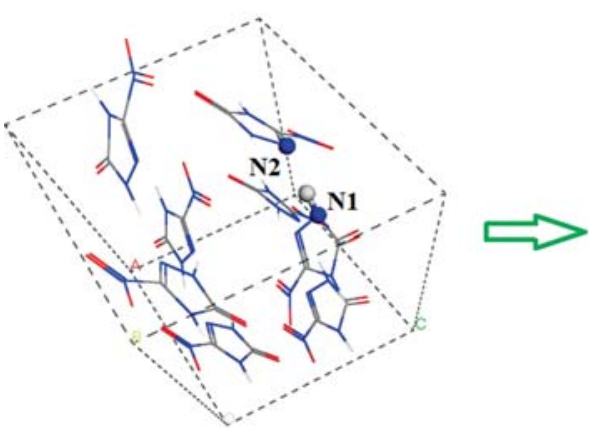

(d)

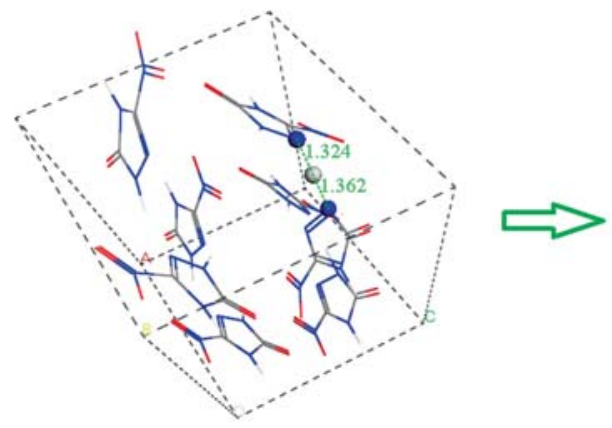

occurring<smiles>O=c1[nH]nc([N+](=O)[O-])[nH]c1=O</smiles>

Figure 8. Snapshots before, during, and after the initiation of the decomposition of NTO through N-H $\cdots \mathrm{O}$ (a and $\mathbf{b})$ and $\mathrm{N} \cdots \mathrm{H} \cdots \mathrm{N}(\mathbf{c}$ and d) intermolecular hydrogen transfer. Nitrogen, oxygen, and hydrogen atoms are represented by blue, red, and white spheres, respectively. 

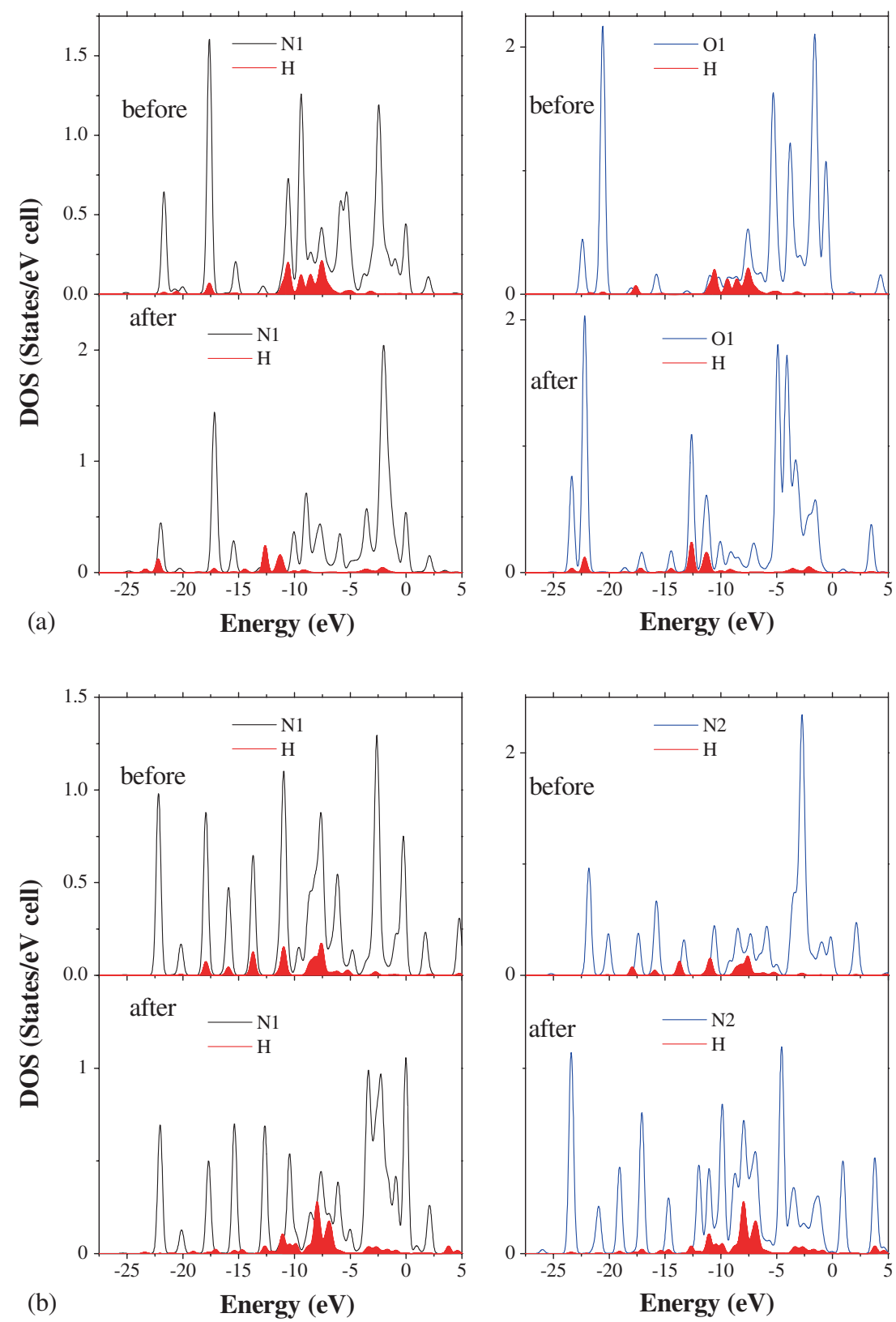

Figure 9. The changing process of the PDOS of the atoms involved in the (a) $\mathrm{N} \cdots \mathrm{H} \cdots \mathrm{O}$ and (b) $\mathrm{N} \cdots \mathrm{H} \cdot \cdots \mathrm{N}$ intermolecular hydrogen transfers in NTO crystal.

Overall, NTO decomposed before melting at the five investigated pressures and two different bimolecular types of initial decomposition mechanisms were observed.

3.3c Decomposition products: Table 4 lists the first decomposition product of NTO under thermal decomposition temperature at different pressures. It is found that the first product is different at different pressures. The first product is $\mathrm{N}_{2}$ at 1 and $2 \mathrm{GPa}, \mathrm{H}_{2} \mathrm{O}$ at $3 \mathrm{GPa}$, or $\mathrm{NO}_{2}$ at 4 and $5 \mathrm{GPa}$. The formation processes of $\mathrm{N}_{2}, \mathrm{H}_{2} \mathrm{O}$, and $\mathrm{NO}_{2}$ are displayed in figure 10. In figure
$10 \mathrm{a}$, one hydrogen atom captures one oxygen atom in the nitro group of one INT molecule to form one $\mathrm{OH}$ and one new INT. This new INT is very unstable and further decomposes to release one $\mathrm{N}_{2}$ molecule through fast and continuous $\mathrm{C}-\mathrm{N}$ breaking, twice, in the ring. In figure $10 \mathrm{~b}$, one hydrogen atom captures one oxygen atom of the nitro group to form one $\mathrm{OH}$ and one new INT. The generated $\mathrm{OH}$ is active and further combines with another hydrogen to form $\mathrm{H}_{2} \mathrm{O}$. In figure 10c, one $\mathrm{OH}$ attacks the $\mathrm{C}$ atom in the ring to form a new $\mathrm{C}$ $\mathrm{OH}$ bond. This process destroys the stability of the ring leading to ring breaking and formation of one $\mathrm{C}-\mathrm{N}$ bond 
(a)
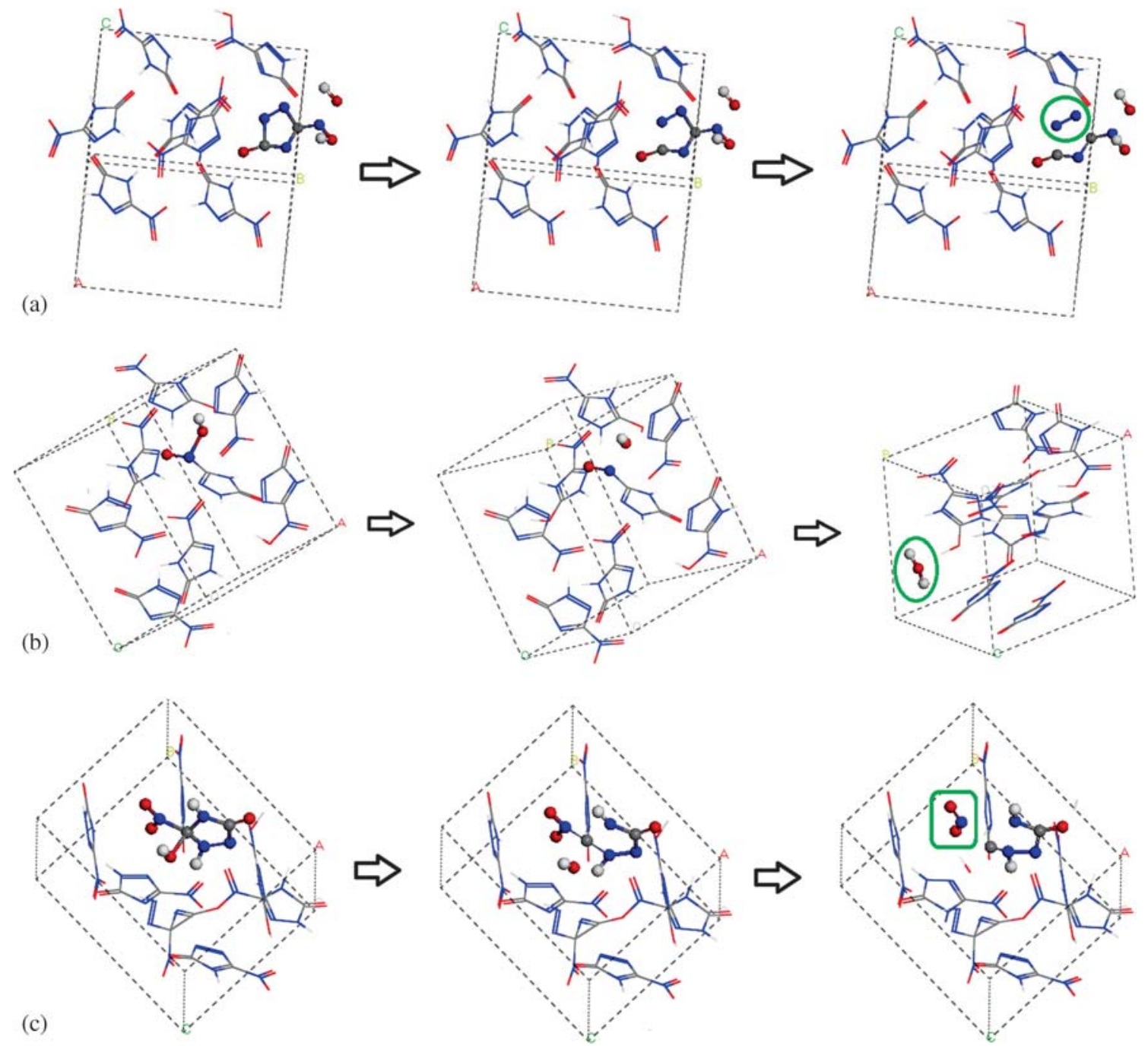

Figure 10. The formation processes of first $\mathrm{N}_{2}, \mathrm{H}_{2} \mathrm{O}$ and $\mathrm{NO}_{2}$ in NTO Crystal. Carbon, nitrogen, oxygen, and hydrogen atoms are represented by gray, blue, red, and white spheres, respectively.

which in turn results in the formation of a new catenulate INT. This new INT is unstable and decomposes to release one $\mathrm{NO}_{2}$ by the $\mathrm{C}-\mathrm{NO}_{2}$ bond cleavage. This was the case with PETN as well. Since our investigated temperature $(531 \mathrm{~K})$ is low, the decomposition degrees of NTO at high pressure are limited; only several products and INTs produced.

To summarize, at thermal decomposition temperature, NTO decomposed before melting at 1,2,3, 4, and $5 \mathrm{GPa}$. This is different from that of PETN. The initial decomposition mechanism was dependent on the pressure. At 1, 2, and $3 \mathrm{GPa}$, the initial decomposition mechanism is the $\mathrm{N}-\mathrm{H}$. . O O intermolecular hydrogen transfer, while at 4 and $5 \mathrm{GPa}$, this is the $\mathrm{N}-\mathrm{H} \cdots \mathrm{N}$ intermolecular hydrogen transfer. The first product was also dependent on the pressure. The first product is $\mathrm{N}_{2}$ at 1 and $2 \mathrm{GPa}, \mathrm{H}_{2} \mathrm{O}$ at $3 \mathrm{GPa}$, or $\mathrm{NO}_{2}$ at 4 and $5 \mathrm{GPa}$.

\section{Conclusions}

In this work, we performed AIMD simulations to study the initial decomposition mechanisms and products of two molecular crystal explosives PETN and NTO under thermal decomposition temperature ( $475 \mathrm{~K}$ for PETN and $531 \mathrm{~K}$ for NTO) coupled with different pressures (1-5 GPa). The pressure effects on the initial decomposition steps and first products on PETN and NTO were very different. PETN melted without further decomposition at $1 \mathrm{GPa}$ but melted and decomposed at 2-5 GPa. It was triggered by $\mathrm{C}-\mathrm{H} \cdots \mathrm{O}$ intermolecular hydrogen transfer and the generated first product is $\mathrm{NO}_{2}$. The initial decomposition and first product were independent of the pressure. NTO decomposed before melting and two different initial decompositions were found. At 1, 2 and $3 \mathrm{GPa}$, the initial decomposition mechanism is the 
$\mathrm{N}-\mathrm{H}$. . O intermolecular hydrogen transfer, while at 4 and $5 \mathrm{GPa}$, this is the $\mathrm{N}-\mathrm{H} \cdots \mathrm{N}$ intermolecular hydrogen transfer. The initial decomposition and generated first product were dependent on the pressure. The first product is $\mathrm{N}_{2}$ at 1 and $2 \mathrm{GPa}, \mathrm{H}_{2} \mathrm{O}$ at $3 \mathrm{GPa}$, and $\mathrm{NO}_{2}$ at 4 and $5 \mathrm{GPa}$. Our study may provide new insights into the initial decompositions of molecular crystal explosives under thermal decomposition temperature coupled with different pressures in atomic details.

\section{Acknowledgments}

This work was supported by the National Natural Science Foundation of China (Grant No. 21273115), the NSAF Foundation of National Natural Science Foundation of China and China Academy of Engineering Physics (Grant No. U12301020), and a Project Funded by the Priority Academic Program Development of Jiangsu Higher Education Institutions.

\section{References}

1. Zhurova E A, Stash A I, Tsirelson V G, Zhurov V V, Bartashevich E V, Potemkin V A and Pinkerton A A 2006 J. Am. Chem. Soc. 12814728
2. Booth A D and Llewellyn F J 1947 J. Chem. Soc. 837

3. Trotter J 1963 Acta Crystallogr. 16698

4. Dreger Z A and Gupta Y M 2013 J. Phys. Chem. A 117 5306

5. Yamawaki H, Fujihisa H, Wakabayashi K, Honda K and Gotoh Y 2013 Propellants Explos. Pyrotech. 38 394

6. Bolotina N B, Zhurova E A and Pinkerton A A $2003 \mathrm{~J}$. Appl. Cryst. 36280

7. Ritchie J P 1989 J. Org. Chem. 543553

8. Sinditskii V P, Smirnov S P and Egorshev V Y 2007 Propellants Explos. Pyrotech. 32277

9. Segall M D, Lindan P J D, Probert M J, Pickard C J, Hasnip P J, Clarkv S J and Payne M C 2002 J. Phys.: Condens. Matter 142717

10. Vanderbilt D 1990 Phys. Rev. B 417892

11. Perdew J P, Burke K and Ernzerhof M 1996 Phys. Rev. Lett. 773865

12. Tkatchenko A and Scheffler M 2009 Phys. Rev. Lett. 102 073005

13. Grimme S 2006 J. Comput. Chem. 271787

14. Nosé S 1984 J. Chem. Phys. 81511

15. Andersen H C 1980 J. Chem. Phys. 722384

16. Wu C J, Fried L E, Yang L H, Goldman N and Bastea S 2009 Nat. Chem. 157

17. Pozzo M, Davies C, Gubbins D and Alfè D 2012 Nature 485355

18. Tsyshevsky R V, Sharia O and Kuklja M M $2013 \mathrm{~J}$. Phys. Chem. C 11718144 